pp 447-471. (C) The Author(s) 2020. This is an Open Access article, distributed under the terms of the Creative Commons Attribution licence (http://creativecommons.org/licenses/by/4.0/), which permits unrestricted re-use, distribution, and reproduction in any medium, provided the original work is properly cited.

doi:10.1017/aer.2019.160

\title{
Evaluating the impact of new aircraft separation minima on available airspace capacity and arrival time delay
}

\author{
E. Itoh $(\mathbb{D}$ \\ eri@mpat.go.jp
}

Air Traffic Management Department

National Institute of Maritime, Port and Aviation Technology

Electronic Navigation Research Institute

Tokyo

Japan

\section{Mitici}

Faculty of Aerospace Engineering

Delft University of Technology

Delft

The Netherlands

\section{ABSTRACT}

Although the application of new, reduced aircraft separation minima can directly increase runway throughput, the impact thereof on the traffic flow of aircraft arriving at the destination airport has not been discussed yet. This paper proposes a data-driven and queue-based modeling approach and presents an analysis of the impact on the delay time of arriving aircraft in the airspace within a radius of 100 nautical miles around an airport. The parameters of our queuing model were estimated by analysing the data contained in the radar tracks and flight plans for flights that arrived at Tokyo International Airport during the 2 years of 2016 and 2017. The results clarified the best arrival strategy according to the distance from the arrival airport: The combination of airspace capacity control and reduction of the flight time and separation variance is the most powerful solution to mitigate delays experienced by arriving traffic while also allowing an increase in the amount of arrival traffic. The application of new wake vortex categories would enable us to increase the arrival traffic to $120 \%$. In addition, the arrival delay time could be minimised by implementing the proposed arrival traffic strategies together with automation support for air traffic controllers. 
Keywords: queuing theory; data analysis; data-driven air transport; arrival management; wake vortex categories

\section{NOMENCLATURE}

$\begin{array}{ll}\text { AMAN } & \text { arrival management } \\ \text { ATM } & \text { air traffic management } \\ C_{A_{i}} & \text { coefficient of variation of aircraft inter-arrival times in airspace area } i \\ C_{B_{i}} & \text { coefficient of variation of aircraft service times in airspace area } i \\ c_{i} & \text { number of servers in airspace area } i \\ D_{i} & \text { delay an aircraft experiences in airspace area } i \\ \text { E-AMAN } & \text { extended arrival management } \\ \text { FCFS } & \text { first come first served } \\ \text { GIM-S } & \text { ground-based interval management spacing } \\ \text { IAT } & \text { inter-aircraft time } \\ i & \text { number of airspace area } \\ \text { ICAO } & \text { international civil aviation organization } \\ \text { IM } & \text { interval management } \\ \text { LRATFM } & \text { long range air traffic flow management } \\ \text { MTOM } & \text { maximum take-off mass } \\ \text { NM } & \text { nautical mile } \\ \text { RECAT } & \text { wake turbulence re-categorization } \\ \text { STD } & \text { standard deviation } \\ \text { TBFM } & \text { time-based flow management } \\ \text { TMA } & \text { traffic management advisory } \\ \text { TRACON } & \text { terminal radar approach control } \\ \text { TSAS } & \text { terminal sequencing and spacing } \\ t_{I A T} & \text { IAT in seconds } \\ \text { WS } & \text { wind span } \\ & \end{array}$

\section{Greek Symbol}

$\mathbb{E}\left[A_{i}\right] \quad$ mean of aircraft inter-arrival times in seconds

$\mathbb{E}\left[B_{i}\right] \quad$ mean of aircraft service times in seconds

$\mathbb{E}\left[D_{i}^{G / G / c}\right]$ expected total airborne delay of a $G / G / c$ queuing model in airspace area $i$ in seconds

$\mathbb{E}\left[D_{i}^{M / M / c}\right]$ expected total airborne delay of a $M / M / c$ queuing model in airspace area $i$ in seconds

$\mathbb{E}\left[t_{\text {min }}\right] \quad$ mean of the minimum time separation in seconds

$\lambda_{i} \quad$ arrival rate (number of arrival aircraft per hour) at airspace area $i$

$\lambda_{R W 22}$ arrival rate (number of arrival aircraft per hour) at Runway 22

$\mu_{i} \quad$ service ratio at airspace area $i$ 


$\begin{array}{ll}\rho_{i} & \text { parameter to estimate queue-stability at airspace area } i \\ \sigma_{A_{i}} & \text { variance of aircraft inter-arrival times in seconds } \\ \sigma_{B_{i}} & \text { variance of aircraft service times in seconds }\end{array}$

\subsection{INTRODUCTION}

Increasing the runway throughput is one of the most promising solutions to accommodate future air traffic growth. In this regard, the application of new wake vortex categories that lower the separation minima between arriving aircraft have been considered ${ }^{(1-3)}$. Consequently, this affects aircraft arrival flows in that these separation minima lead to a reduction in the aircraft inter-arrival time, or, equivalently, they allow an increased aircraft arrival rate at an airport. An analysis is required to clarify whether the use of these new separation minima standards contributes to efficiently controlled arrival traffic, particularly for increased, future air traffic volumes. Further, it would be of interest to analyse the impact of these new minima separation standards on the airspace surrounding the destination airport, given the available airspace capacity, while ensuring reasonable delays in arrival times. To clarify this, we propose a new approach based on data-driven queuing models to analyse the impact of new aircraft separation minima on the traffic flow of arrival aircraft. For our analysis, we consider the airspace within a radius of 100 nautical miles (NM) from the destination airport. We demonstrate the effectiveness of our models with a case study at Tokyo International Airport, the fifth busiest airport in the world ${ }^{(4)}$.

The management of aircraft arrivals at airports is central to airport operations. In the United States, the Traffic Management Advisory (TMA) ${ }^{(5)}$ was deployed in air traffic control centers in the 1990s. The enhanced version thereof, Time-Based Flow Management (TBFM) ${ }^{(6)}$ and Terminal Sequencing and Spacing (TSAS) ${ }^{(7)}$, considers future airborne-based operations ${ }^{(8)}$. These systems contribute to sequencing and time spacing the arrival traffic consistently in en-route and terminal airspace areas. In Europe, the on-going SESAR project has facilitated collaboration among European countries and contributed to the development of 'Enhanced' Arrival Management (AMAN), which coordinates the arrival time-schedules covering wider ranges of airspace than in the case of conventional operations ${ }^{(9)}$. In the Asia-Pacific region, strategic air traffic flow management is achieved by using Long Range Air Traffic Flow Management (LRATFM), which has been devised to provide a basis for research into applications beyond the time-frames of the current system ${ }^{(10)}$. On-going Japanese research and development on an 'Extended' AMAN (E-AMAN) aims to ensure the efficiency of the traffic flow arriving at Tokyo International Airport ${ }^{(11)}$. In the E-AMAN scheme, arrival traffic flow control, which is designed to coordinate the traffic volume by considering the limited airspace capacity and runway throughput, shifts to time-based operations close to the destination airports, which ensures minimum time-spacing between arrivals. One of the most important requirements E-AMAN is designed to comply with, is to schedule the arrival time to accommodate an increasing arrival ratio, while reducing the arrival time delay.

Several studies have analysed the aircraft arrival process at airports by means of queuing theory. For example, the aircraft arrival delay was analysed ${ }^{(12-15)}$ by employing queuing models focused especially on runway-related delay and capacity constraints. Bauerle et $\mathrm{al}^{(12)}$ determined the expected waiting time for aircraft arriving at a single runway using an $M / S M / 1$ queuing model. Simple bounds for the expected waiting time were determined in comparison to $M / G / 1$ and $M / D / 1$ models. However, in their case study, the authors did not make use of operational data or compare their results against actual data. Rue 
and Rosenshine ${ }^{(14)}$ modeled the arrival of aircraft at Pittsburgh International Airport as an $M / M / 1$ and $M / E_{k} / 1$ queue. The authors determined, using Semi-Markov decision processes, the maximum number of aircraft allowed to land at the airport during peak hours. Bolender and Slater ${ }^{(15)}$ estimated the expected aircraft arrival delay by means of $M / D / n$ queues, which yielded reasonable results when compared with numerical simulation data. Again, no comparative analysis with actual flight data was conducted. Long et al. ${ }^{(13)}$ modeled the National Airspace System in the United States as a network of aircraft arriving, taxiing, and departing queues, with the TRACON sectors modeled as an $M / E_{3} / n$ queue. The study provided a macroscopic view of the impact of ATM technologies and costs. Our previous paper ${ }^{(16)}$ presented an analysis of the arrival delay at an airport using a $G / G / c$ queuing model. In this study, we considered extending the arrival airspace, while considering current minimum separation standards and airspace capacity. In another study ${ }^{(17)}$ we evaluated different tactical control strategies for arrivals at an airport using a $M / G / c / K$ queuing model. In this paper, we present an evaluation of the impact of new separation minima standards on arrival delay. We model the traffic flow of aircraft arriving from within a radius of $100 \mathrm{NM}$ from the destination airport by means of a $G / G / c$ queue, and provide a microscopic view of the analysis to clarify the optimal balance between increasing the arrival rate considering the airspace capacity, with the ultimate aim of minimising the delay in arrival time.

This remainder of the paper is organised as follows. Section 2 introduces air traffic operations at Tokyo International Airport and characterises the arrival traffic data recorded in 2016 and 2017. This data analysis supports the models that are subsequently presented. Section 3 compares the potential increase in the runway throughput by applying new wake vortex categories, RECAT-Dubai, EU, US 1.5, with a current standard. The expected future arrival ratio is estimated by combining these categories and safety margins depending on the levels of automation support that were introduced for air traffic controllers. Section 4 proposes a queue-based modeling approach in which the aircraft arrival process is formulated as a $G / G / c$ queuing model. Based on the data-driven analysis, the probability distributions of the interarrival time and service time are estimated. The number of virtual service counters, which directly translates into capacities of the assigned airspace, is also estimated based on the traffic data. In Section 5, the $G / G / c$ queuing model is applied to estimate the aircraft arrival delay time as a function of the distance from the destination airport by increasing the radius of the airspace centered at the arrival airport from 10 to $100 \mathrm{NM}$ in increments of $10 \mathrm{NM}$. We analyse the positive impact of reducing the inter-arrival time within the arrival traffic flow on the airspace capacity. In this context, the aircraft delay time is estimated by means of the proposed queue-based approach. Section 6 discusses the impact of new aircraft separation minima on the traffic flow of aircraft arriving at the airport. Furthermore, the optimal arrival strategy, which meets the key requirements of the E-AMAN design, is suggested. This strategy mitigates the arrival delay time while increasing the arrival traffic ratio. Finally, Section 7 provides the conclusions and outlines our plans for future work.

\subsection{CASE STUDY DATA DESCRIPTION - TOKYO INTERNATIONAL AIRPORT}

\subsection{Aircraft arrival operations}

In this section we describe the aircraft arrival operations at Tokyo International Airport. Tokyo International Airport is the fifth busiest airport in the world with respect to passenger traffic, 


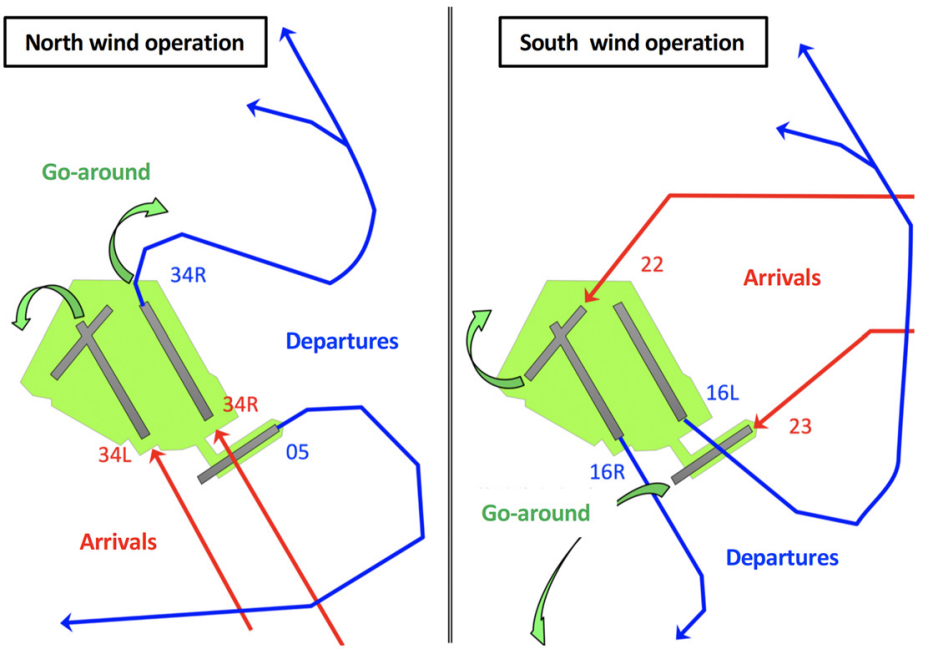

Figure 1. Departure and arrival operations depending on wind direction.

processing a total of $83,189,933$ passengers in 2017 . An increase of $4.32 \%$ in the number of passengers was registered in 2017 relative to $2016^{(4)}$. The maximum number of departures and arrivals accommodated per year is 447,000, with a maximum of 80 operations per hour. Over $60 \%$ of the domestic flights in Japan are concentrated at this airport. The airport makes use of four runways on a daily basis, with the choice of runway configuration depending on the wind direction (see Fig. 1). For operations at times of a northerly wind, runway 34L is used for arrivals from the south-west direction, whereas runway 34R is used for arrivals from the north. Runway 34R is also used for departure traffic. For operations when a southerly wind prevails, runway 22 is used for arrivals from the south-west, and runway 23 for arrivals from the north.

In this study we consider flight plans and track data for a period of 71 days, which are randomly selected from the odd months of 2016 and 2017. In 2016, there were a total average of 608 arrivals per day, of which 530 flights were domestic and 78 flights international. In 2017, eight additional international flights were accommodated every day compared to 2016 . Thus, in 2017, there were a total average of 614 arrivals per day, of which 530 flights were domestic and 84 flights were international. The total number of arrivals between 8:00AM and 11:00PM is slightly below the maximum allowed daily traffic threshold, and the most congested period is between 5:00PM and 10:00PM.

\subsection{Data-driven analysis of the aircraft arrival flows}

One of the features of the arrival traffic flow at Tokyo International Airport is that the arrivals are clustered either in the northern or the southwestern part of the airport. The period 8:00AM-11:00PM has a total of 569 arrivals, of which 422 flights arrive from the south-west, and 147 flights arrive from the north. Figure 2 shows the flight track data for one day of operation in September 2017. The flight tracks of arrivals that use runway 22 are drawn in red, whereas arrivals at runway 23 are drawn in blue.

Air traffic flow in Japan is strategically controlled with a central focus on arrivals at Tokyo International Airport. Figure 3 shows the number of arrival flights crossing the concentric circles with radii ranging from 10 to $100 \mathrm{NM}$ every $10 \mathrm{NM}$ centered at Tokyo International 


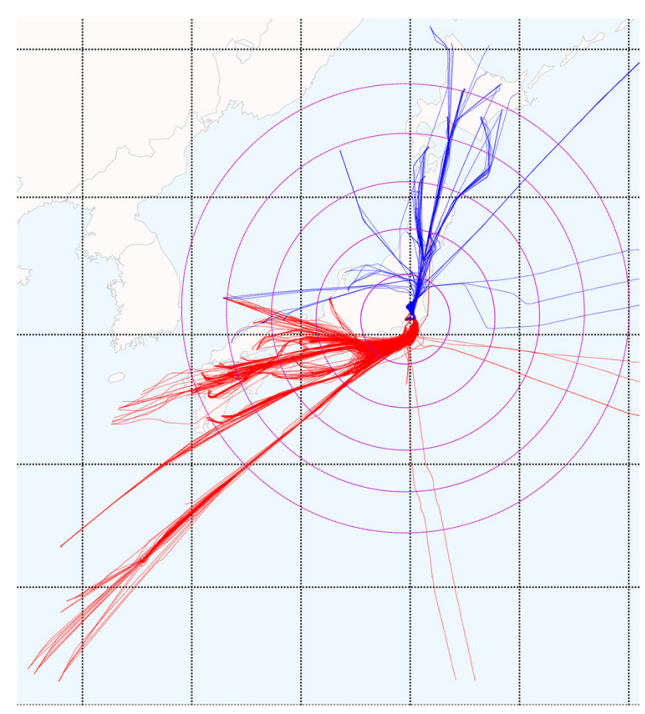

Figure 2. Example of flight tracks during an entire day in September 2017. The red and blue tracks represent the traffic flow from the south-west and the north, respectively. Concentric circles are drawn every 100NM for the airspace with a radius from 100 to 500NM centered at Tokyo International Airport.

Airport per hour from 5:00PM to 10:00PM. The total number of flight arrivals is controlled within a maximum of 40 (30 arrivals from the south-west and 10 arrivals from the north). Figure 4 shows the distribution of departure airports for both domestic and international flights depending on the number of arrivals in a day. It is obvious that a larger number of domestic flights arrive from the south-west. Additionally, international arrivals from airports in other Asian countries also approach from the south-west. As global air traffic passenger demand is expected to increase by $250 \%$ in the next 20 years according to Asian economic growth forecasts ${ }^{(18)}$, a further increase in arrivals from the south-west is estimated to occur.

Table 1 shows the types of aircraft, expressed as a percentage, that arrive and depart. The majority of the aircraft are used for short and medium-distance passenger transportation using A737-800 (B738), A320, and B767-300 (B763). The second category includes long-distance passenger aircraft such as B777-200 (B772) and B787-8 (B788). Finally, less than 1\% of the flights are business jets including Gulfstream and Bombardier products.

\subsection{CURRENT AND NEW AIRCRAFT SEPARATION MINIMA}

\subsection{Wake vortex categories}

The minimum aircraft inter-arrival time is decided based on wake vortex categories, which define aircraft separation minima between the preceding and succeeding aircraft according to their aircraft type. Table 2 compares the aircraft types by categorising them according to the maximum take-off mass (MTOM) and length of wind span (WS) of the aircraft based on four different standards: 'Current ICAO' is the current international standard, 'RECAT-Dubai' is the new standard implemented at Dubai International Airport ${ }^{(3)}$, 'RECAT-US 1.5' is the new 


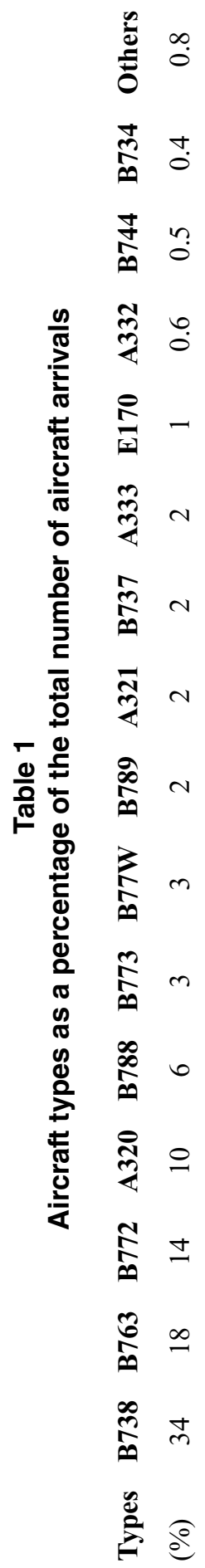




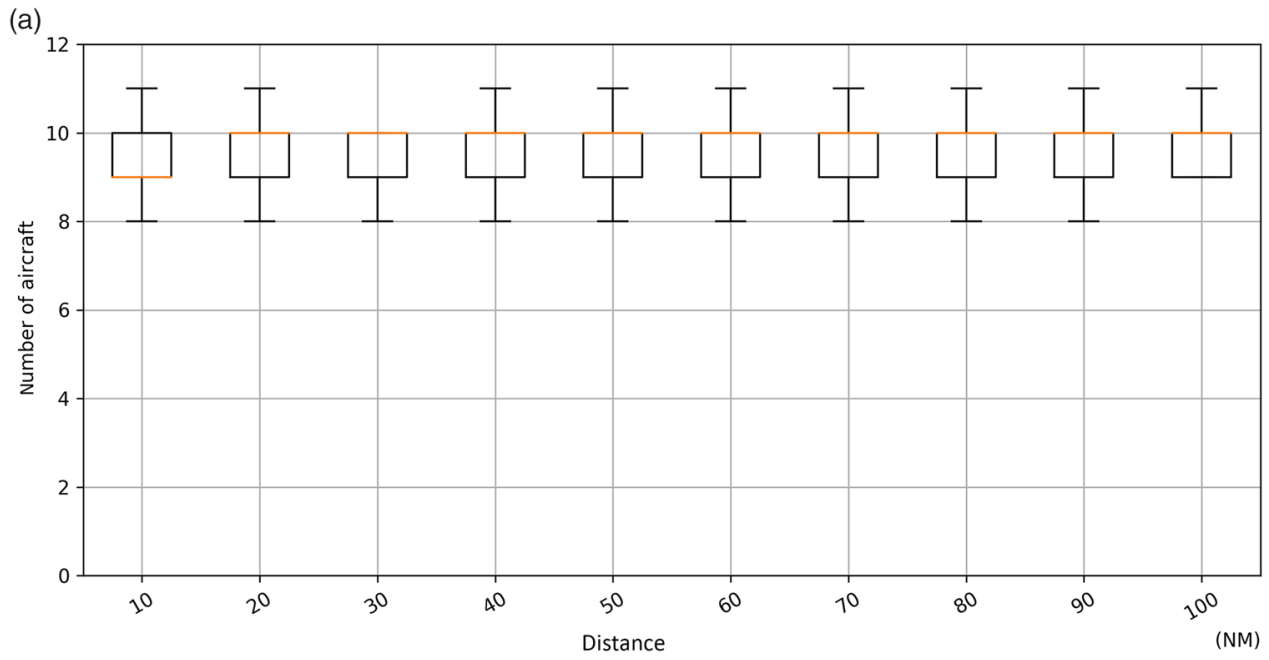

(b)

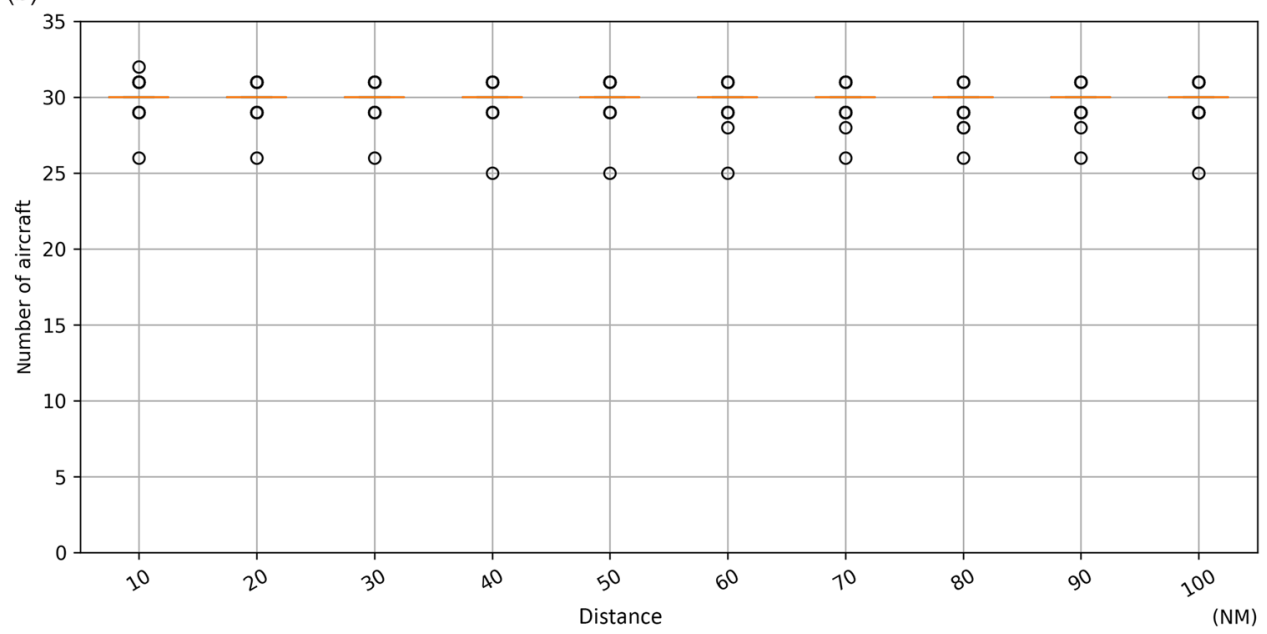

Figure 3. Number of aircraft arriving per hour during the hours 5:00PM-10:00PM in 2016 and 2017. (a) Traffic flow from the north. (b) Traffic flow from the south.

US standard ${ }^{(2)}$, and 'RECAT-EU' is the new EU standard ${ }^{(1)}$. In these standards ${ }^{(1-3)}$, the minimum distance separation is decided using combinations of preceding and succeeding aircraft types. Specifically, 'RECAT-Dubai' employs the combination of low separation minima in 'RECAT-US1.5' and 'RECAT-EU,' and is proposed as the new ICAO standard. Thus, the aircraft types arriving at a destination airport (see Table 1) determine the runway throughput following these separation standards.

\subsection{Distance separation minima under RECAT-Dubai}

Table 3 summarises the distance-based wake turbulence separation minima based on the RECAT-Dubai standards ${ }^{(3)}$. The radar separation minimum (3NM in Japan) is applied for category sets not included in the table. Figure 5 shows the frequency of a specific type of 


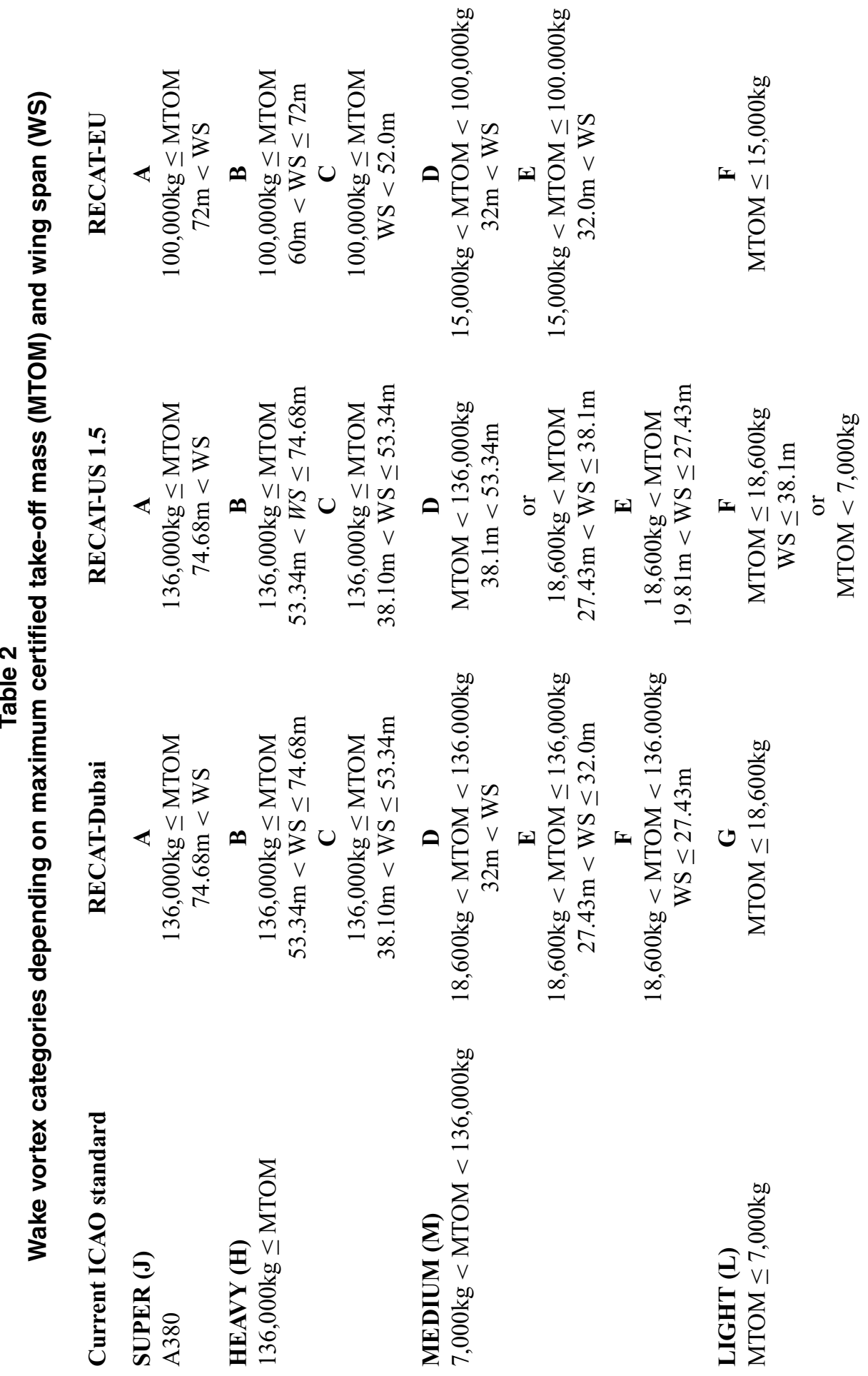


(a)

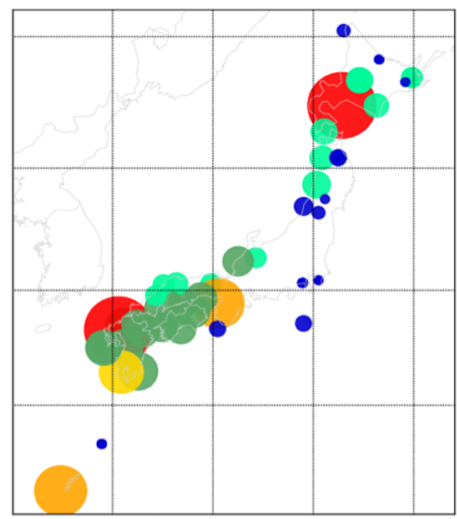

(b)

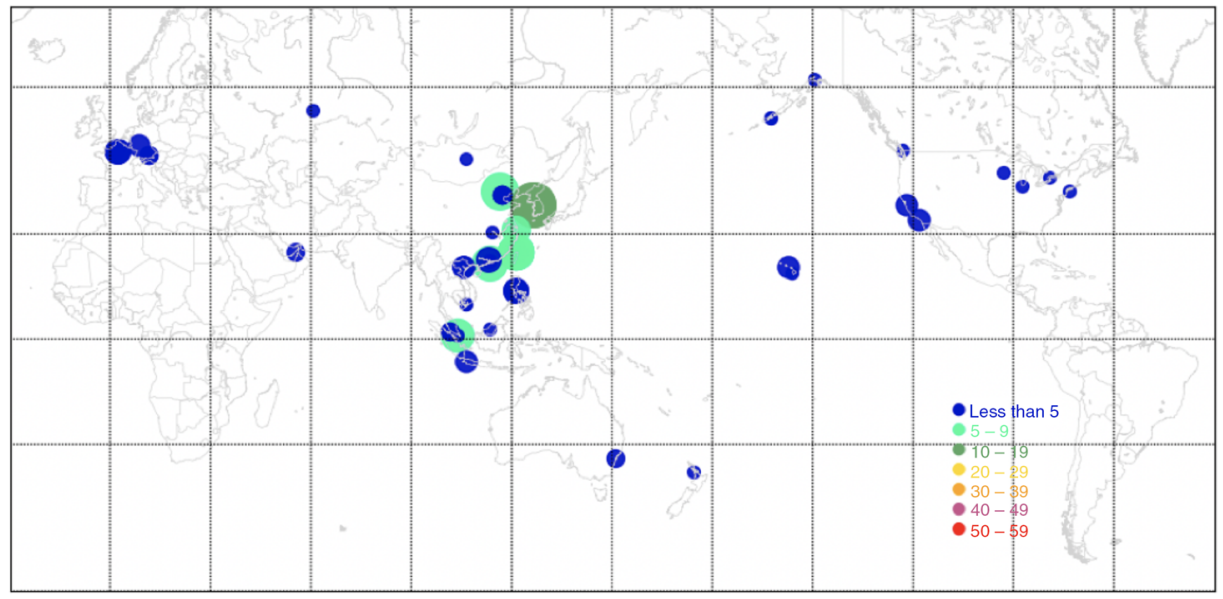

Figure 4. Distributions of departure airports depending on the numbers of arrivals in a day. (a) Departure airports of domestic flights. (b) Departure airports of international flights.

preceding-succeeding aircraft set when the RECAT-Dubai standard is applied to current traffic flow arriving at Runways 22 and 34L between 8:00AM and 10:00PM. Arrival sequencing is determined by the radar data. The majority of aircraft (see Table 1) arriving at Tokyo International Airport are of types $\mathrm{D}, \mathrm{B}$, and $\mathrm{C}$ with a ratio of approximately 5:3:2. When arrival sequencing is optimised by means of, for instance position shifting, one or two additional aircraft arrivals per hour are accommodated, relative to the current first come first served (FCFS) sequencing. However, these position-shifting operations are not necessarily equitable from the airlines' point of view. Furthermore, it is not clear how such optimisation operations can be achieved without increasing the workload of air traffic controllers and/or without any automation support for the air traffic controllers. Equally important, these optimisation operations may also propagate traffic congestion to succeeding and/or surrounding aircraft traffic. For these reasons, the optimisation of arrival sequencing was not addressed in the study presented in this paper. 
Table 3

\section{Distance-based wake turbulence separation minima under RECAT-Dubai} separation standards

\section{Preceding aircraft group}

A

B

$\mathrm{C}$

D

E

\author{
Succeeding \\ aircraft group
}

B

C, D

E, F

G

B

C, D

E, F

G

D

E, F

G

G

G
Distance-based

wake turbulence

separation minima

$4.0 \mathrm{NM}$

$5.0 \mathrm{NM}$

$6.0 \mathrm{NM}$

$8.0 \mathrm{NM}$

$3.0 \mathrm{NM}$

$4.0 \mathrm{NM}$

$5.0 \mathrm{NM}$

$7.0 \mathrm{NM}$

$3.0 \mathrm{NM}$

$3.5 \mathrm{NM}$

$6.0 \mathrm{NM}$

4.0NM

4.0NM

(a)

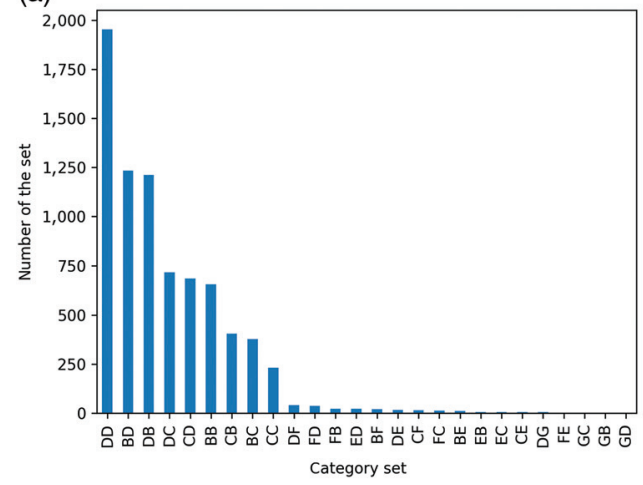

(b)

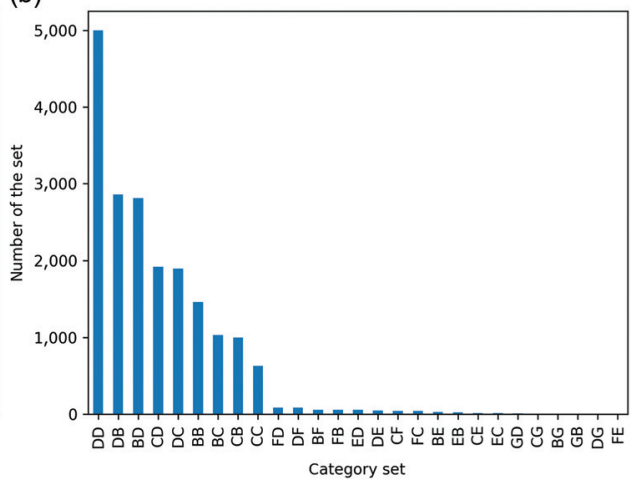

Figure 5. Current arrival sets of aircraft categories according to RECAT-Dubai standards. (a) Runway 22, southerly wind operation, 8-22h. (b) Runway 34L, northerly wind operation, 8-22h.

\subsection{Aircraft time separation minima under different standards}

In this section we determine the time separation associated with the current ICAO, RECATDubai, RECAT-US 1.5, and RECAT-EU standards. The same arrival sequences are given to estimate the time separation. Figure 6 shows the minimum time separation, when these four standards are applied to the arrival traffic flow at runway 22 and runway 34L. We note that these runways are used only for arrivals during the congested time period 5:00PM-10:00PM at Tokyo International Airport. To determine the aircraft time-based separation for each of the four standards, the distance-based separation minima are divided by the aircraft ground speed 


\section{Table 4}

Comparing the mean and STD of the arrival time separation

\section{Current ICAO standard \\ RECAT-Dubai \\ RECAT-US 1.5 \\ RECAT-EU}

Runway 22, southerly wind operation, 5:00PM-10:00PM
Mean (sec)
99.10
84.25
89.61
84.53
STD (sec)
22.50
12.56
20.31

Runway 34L, northerly wind operation, 5:00PM-10:00PM

$\begin{array}{lllll}\text { Mean }(\mathrm{sec}) & 95.75 & 81.80 & 87.03 & 82.15 \\ \text { STD }(\mathrm{sec}) & 21.94 & 12.34 & 19.71 & 12.62\end{array}$

(a)

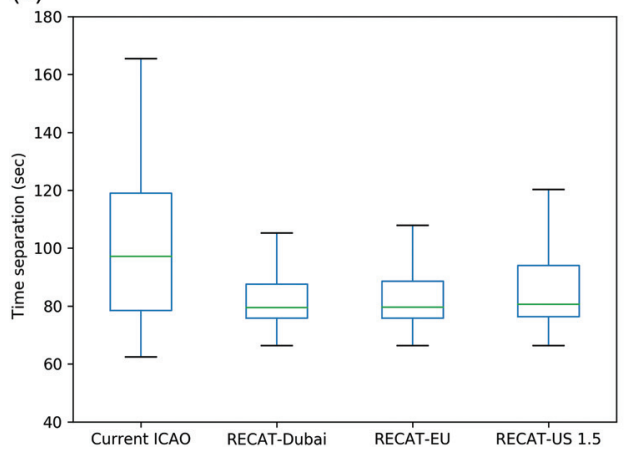

(b)

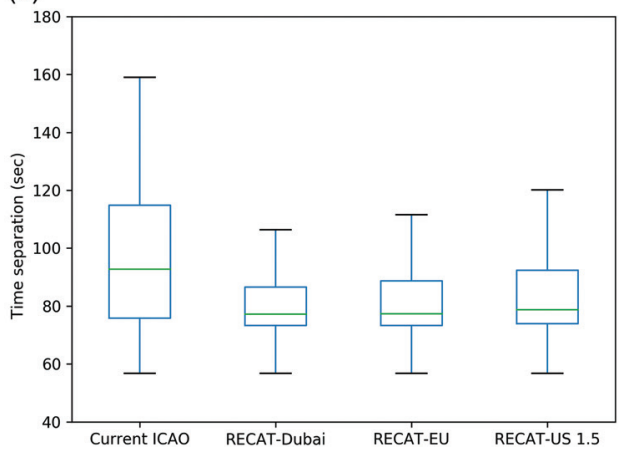

Figure 6. Comparing the time-separation when four different wake vortex categories are applied to the arrivals at Tokyo International Airport. (a) Runway 22, southerly wind operation, 17-22h. (b) Runway 34L, northerly wind operation, $17-22 \mathrm{~h}$.

at $5 \mathrm{NM}$ prior to the runway threshold. We obtained this aircraft speed from the flight track data recorded during the period 2016-2017. Table 4 compares the mean and STD values of the time-based separation when the four standards are taken into consideration. As shown in Fig. 6 and Table 4, RECAT-Dubai has the best potential to minimise aircraft inter-arrival time, whereas RECAT-EU shows slightly larger values. However, it is unrealistic to analyse arrival traffic flows only by considering the time-based separation minima. This is because the air traffic controllers use safety margins, together with the separation minima, to ensure that the separation requirements are always maintained during operations. As such, the next section presents an analysis of the separation minima, together with the safety margins.

\subsection{Safety margins depending on the levels of automation support}

As a benchmark for safety margins, the Inter-Aircraft Time (IAT) was introduced ${ }^{(19)}$. This safety margin corresponds to one standard deviation (STD) value of the aircraft arrival time, i.e. $68 \%$ of the time the aircraft arrives within the mean of the assigned arrival time $\pm 1 / 2$ IAT. The value of IAT depends on the level of automation of the tools that support air traffic controllers, as provided in Table 5: 'No metering' is the baseline capacity, i.e. the amount traffic the air traffic controllers are able to handle without using any automation tools; 'Metering' is the capability when air traffic controllers are supported by arrival management (AMAN) 


\section{Table 5}

Inter-Aircraft Time (IAT) when applying different levels of automation ${ }^{(19)}$

$\begin{array}{lc}\text { Capacity } & \text { IAT (sec) } \\ \text { No metering (baseline) } & 18.0 \\ \text { Metering } & 16.5 \\ \text { GIM-S, TSAS } & 12.0 \\ \text { IM } & 5.0\end{array}$

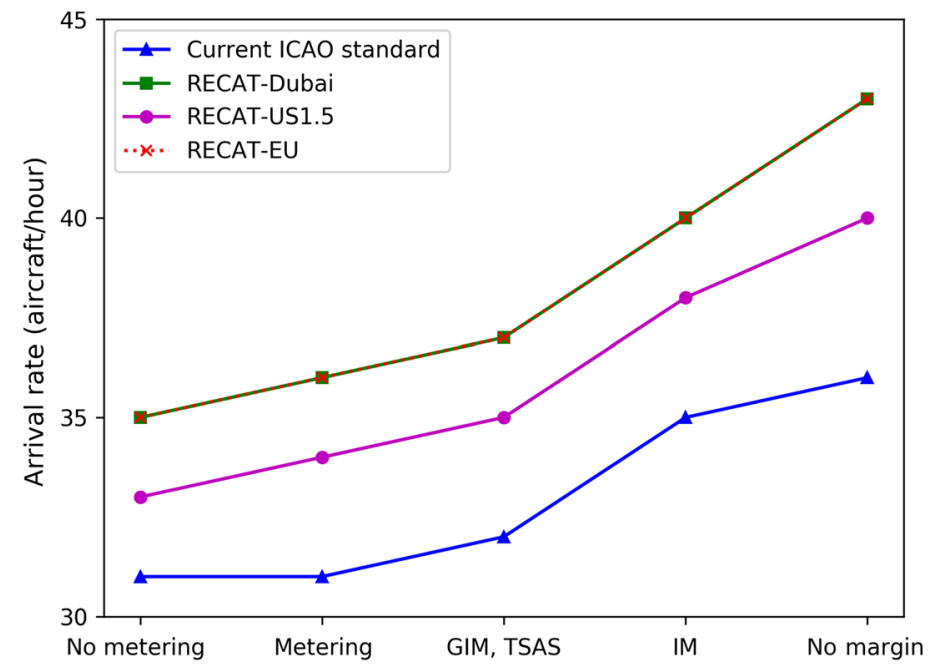

Figure 7. Estimation of arrival rates according to combinations of wake vortex categories and automation levels.

systems, which automatically suggest the ideal arrival time schedules; 'GIM-S, TSAS' is the capability supported by advanced levels of AMAN (refer to Ref. (7)); and 'IM' is its combination with aircraft self-separation (refer to Ref. (8)). In general, as the level of automation support increases, the values of IAT decrease. Therefore, a higher level of automation support can accommodate an increased aircraft arrival rate.

\subsection{Estimating the arrival rate at Runway 22 under various separation standards and safety margins resulting from automation}

Figure 7 shows the arrival rate (number of arrival aircraft per hour) at Runway $22 \lambda_{\mathrm{RW} 22}$, under the four separation standards and considering the safety margins for different levels of automation. We determine this arrival rate as follows:

$$
\lambda_{\mathrm{RW} 22}=\frac{3600.0}{\mathbb{E}\left[t_{\mathrm{min}]}+t_{\mathrm{IAT}}\right.}
$$

where $\mathbb{E}\left[t_{\mathrm{min}}\right]$ is the mean of the minimum time separation (sec) at Runway 22 for southerly wind operation during the period 5:00-10:00PM, corresponding to each wake vortex category in Table 8, and $t_{\mathrm{IAT}}$ is IAT in seconds corresponding to each automation level in Table 5. 
Figure 7 shows that the introduction of the RECAT categories has the potential to support an increase in the aircraft arrival rate up to 133\% (40 arrivals per hour) with the highest automation support 'IM,' when compared with the current arrival rate of 30 arrivals per hour from the south-west (see Fig. 3(b)). When considering the highest level of automation support 'IM,' the current ICAO standard supports an increase in the arrival rate of up to $116 \%$ (35 arrivals per hour). Without any automation support, the current ICAO standard supports an increase in the arrival rate up to $103 \%$ (31 arrivals per hour). However, RECAT-Dubai and RECAT-EU lead to an increase in up to $116 \%$ (35 arrivals per hour) relative to the current arrival rate (30 arrivals per hour). In the case of 'metering' automation support, RECATDubai and RECAT-EU increase the arrival rate up to $120 \%$ (36 arrivals per hour), compared to the current ICAO standards that allow the arrival rate to increase to a maximum rate of $103 \%$ (31 arrivals per hour).

\subsection{MODELING THE FLOW OF ARRIVAL AIR TRAFFIC UNDER CURRENT OPERATIONAL STANDARDS}

\subsection{Modeling the flow of arrival air traffic using a G / G / c queuing model}

We propose a queuing model for traffic arriving from the south-west at Tokyo International Airport between 5:00PM and 10:00PM, which represents the most congested arrival period. We specify our queuing model using the arrival flow, service time, and the number of servers as follows. The aircraft arrive in a given airspace according to an hourly rate, i.e. a specified number of arrivals per hour, referring to Figs 3(b) and 7. The time between two consecutive arrivals in an airspace area is termed the aircraft inter-arrival time. Upon arrival, the aircraft receives a service time, i.e. the time the aircraft spends flying in the given airspace. We consider a multi-server queuing model, in which the number of servers indicates the number of aircraft that are allowed to be present at any time in the given airspace, i.e. the capacity of the given airspace. We apply this model to nine disjoint airspace areas, defined as concentric circles centered at the airport and with radii increasing in increments of 10NM (see Fig. 8).

We formulate this aircraft arrival process by means of a $G / G / c$ queuing model as follows. We consider the circular airspace with a radius of $100 \mathrm{NM}$ centered at the airport. We partition this area using concentric circles (see Fig. 8) centered at the airport, with radii at increments of $10 \mathrm{NM}$. This partitioning defines nine airspace areas $1,2, \ldots, 9$, where area 1 is the airspace area centered at the airport within the circular rings with radii of 10 and $20 \mathrm{NM}$, area 2 is the airspace area centered at the airport within the circular rings with radii of 20 and 30NM radii, and so on. An aircraft arriving at area 1 crosses the circle with radius of 20NM, whereas an aircraft arriving at area 2 crosses the circle with radius of $30 \mathrm{NM}$, and so on.

The $G / G / c$ queuing model allows us to estimate potential aircraft delay time under the assumption that both service time and inter-arrival time follows general distributions. This paper applies the $G / G / c$ model because this model fits the data characteristics of the both distributions of service time and inter-arrival time. An airborne delay would occur when the aircraft is requested to fly a longer route, i.e. vectoring/metering/speed adjustments.

Let $D_{i}$ denote the delay an aircraft experiences in airspace area $i, 1 \leq i \leq 9$ because of the lack of capacity in area $i-1$. Then we approximate the total airborne delay in airspace area $i$ as follows ${ }^{(20-23)}$ :

$$
\mathbb{E}\left[D_{i}^{G / G / c}\right] \simeq \mathbb{E}\left[D_{i}^{M / M / c}\right] \frac{C_{A_{i}}^{2}+C_{B_{i}}^{2}}{2},
$$




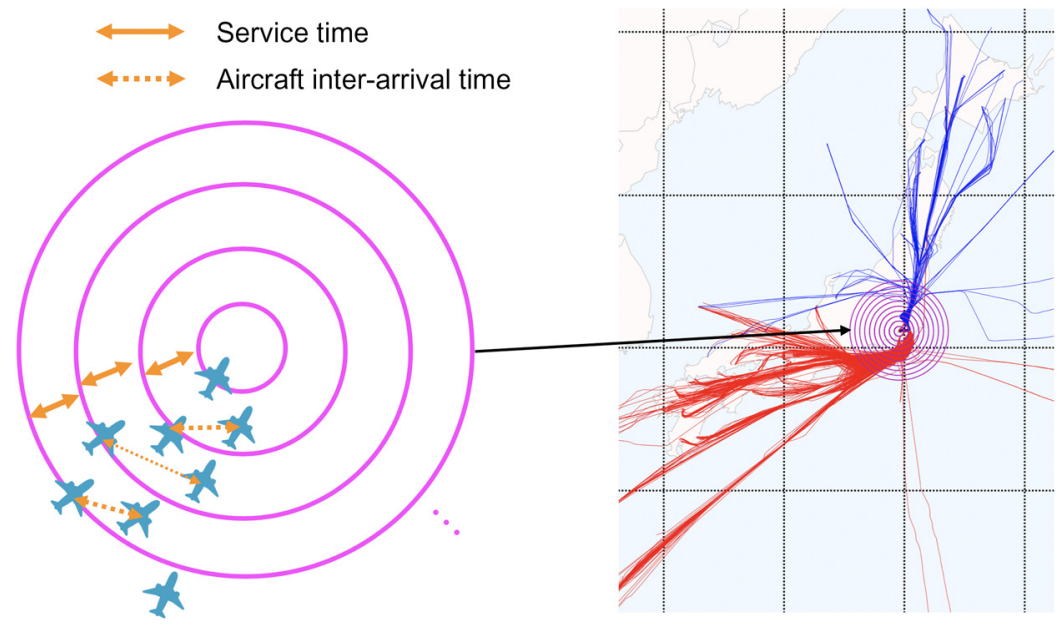

Figure 8. Illustration of the service time and inter-arrival time in the airspace divided into nine areas.

where $C_{A_{i}}=\frac{\sqrt{\sigma\left[A_{i}\right]}}{\mathbb{E}\left[A_{i}\right]}$ is the coefficient of variation of the aircraft inter-arrival time in airspace area $i$ and $C_{B_{i}}=\frac{\sqrt{\sigma\left[B_{i}\right]}}{\mathbb{E}\left[B_{i}\right]}$ is the coefficient of variation of the aircraft service time in airspace area $i$. Here $\mathbb{E}\left[A_{i}\right]$ and $\sigma\left[A_{i}\right]$ are the mean and variance of aircraft inter-arrival time, and $\mathbb{E}\left[B_{i}\right]$ and $\sigma\left[B_{i}\right]$ are the mean and variance of aircraft service time in the airspace area $i$.

In Equation (2), $\mathbb{E}\left[D_{i}^{M / M / c}\right]$ denotes the expected aircraft delay time in airspace area $i$ when a $M / M / c$ queuing model is considered, which is well known ${ }^{(16,17,24,25)}$. This means that the arrival process is considered a Poisson process with a parameter $\lambda_{i}$, with service times are assumed to follow an exponential distribution with a parameter $\mu_{i}$ and there is a fixed number of parallel servers $c_{i}$. Identical servers is well-known and determined, following Refs. (24) and (25).

Moreover, for stability, we have that

$$
\rho_{i}=\frac{\lambda_{i}}{c_{i} \cdot \mu_{i}}<1
$$

If $\rho_{i}$ is not less than 1 , then the queue becomes unstable and the estimated delay time for incoming aircraft tends to become extraordinarily long.

\subsection{Parameter estimation for the G / G / c queuing model based on flight track data analysis}

We estimate the parameter of the $G / G / c$ queuing model as follows. For each airspace area $i \in$ $\{1,2, \ldots, 9\}$, we estimate the values of $c_{i}$ to be the number of aircraft present at the same time in this area, as recorded in the historical flight track data. Figure 9 shows the number of aircraft present in airspace area $i \in\{1,2, \ldots, 9\}$ every 10 min during the time period 5:00PM-10:00PM during the 2 years (2016-2017) of flight track data. An analysis of the historical flight data shows that the highest mean number of aircraft in airspace $i, i \in\{1,2, \ldots, 9\}$ is at most two. Thus, we choose $c=2$ to represent the current capacity for airspace $i, i \in$ $\{1,2, \ldots, 9\}$.

The value $c=2$ is also relevant from the perspective of safety requirements and minimum aircraft separation standards because the inter-arrival aircraft distance is approximately 
Table 6

Parameters of exmpirical distributions of the inter-arrival time and service time in seconds and squared seconds, for the G/G/c model

$\begin{array}{lccccccccc}\boldsymbol{i} & \mathbf{1} & \mathbf{2} & \mathbf{3} & \mathbf{4} & \mathbf{5} & \mathbf{6} & \mathbf{7} & \mathbf{8} & \mathbf{9} \\ \mathbb{E}\left[A_{i}\right] & 111.5 & 112.4 & 111.8 & 108.9 & 108.3 & 107.3 & 106.7 & 106.2 & 106.3 \\ \sigma\left[A_{i}\right] & 473.7 & 623.3 & 707.2 & 2607 & 3488 & 3720 & 4048 & 4267 & 4723 \\ \mathbb{E}\left[B_{i}\right] & 219.7 & 153.7 & 186.0 & 163.2 & 121.9 & 99.24 & 93.18 & 86.11 & 83.84 \\ \sigma\left[B_{i}\right] & 7016 & 508.5 & 3666 & 1640 & 331.9 & 177.1 & 181.6 & 154.5 & 179.5 \\ \rho_{i}(c=2) & 0.9853 & 0.6838 & 0.8313 & 0.7495 & 0.5626 & 0.4624 & 0.4364 & 0.4053 & 0.3944\end{array}$

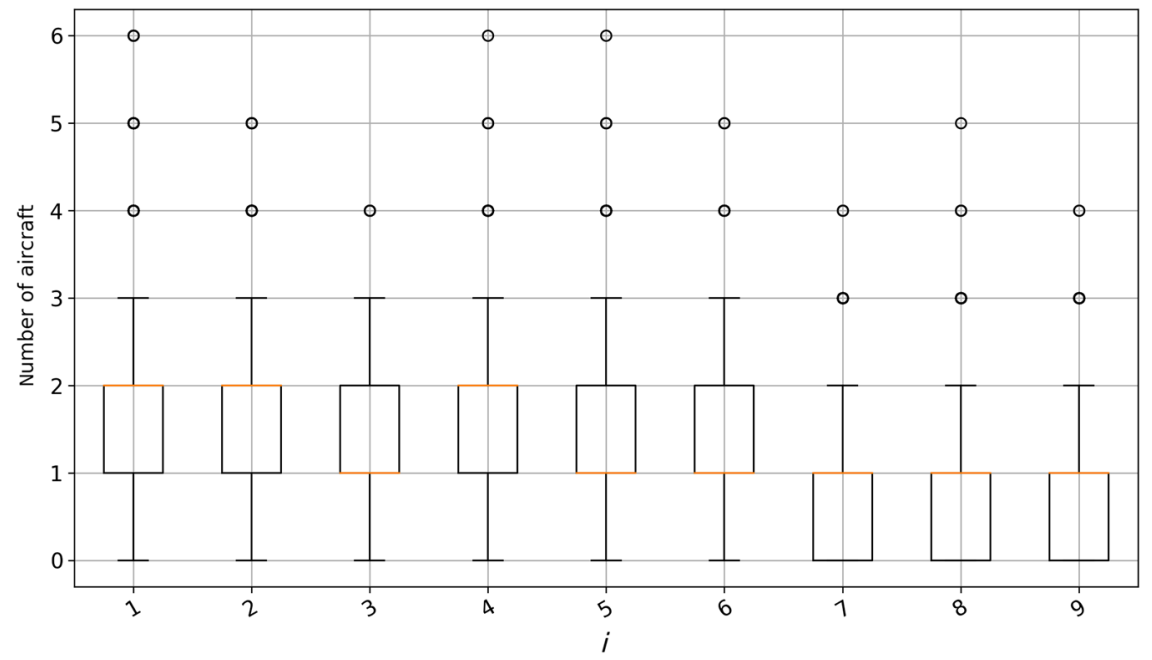

Figure 9. Data-driven estimates of the number of servers, $c$, for the $G / G / c$ model.

5NM (i.e. two aircraft within a 10NM range in-trail), which is exactly the minimum aircraft separation currently required for radar separation in the en-route airspace.

The mean and variance of the inter-arrival time, $\mathbb{E}\left[A_{i}\right]$ and $\sigma\left[A_{i}\right]$, are estimated from the data distribution of the time-separation between the preceding and succeeding aircraft when entering airspace area $i \in\{1,2, \ldots, 9\}$. The mean and variance of the service time, $\mathbb{E}\left[B_{i}\right]$ and $\sigma\left[B_{i}\right]$, are estimated from the data distribution of the flight time in each airspace area $i$. Table 6 lists the values of these parameters with $\rho_{i}$ when $c=2$ is given. As shown in Table 6, all values of $\rho_{i}$ satisfy the stability condition in Equation (3).

\section{ANALYSING THE IMPACT OF NEW AIRCRAFT SEPARATION STANDARDS ON ARRIVAL OPERATIONS}

\subsection{Estimating the minimum required airspace capacity}

As discussed in Section 4.1, Equation (3) shows the condition for queue stability. In this section we estimate the minimum required airspace capacity while ensuring that the queue remains stable, i.e. that $\rho<1$. 


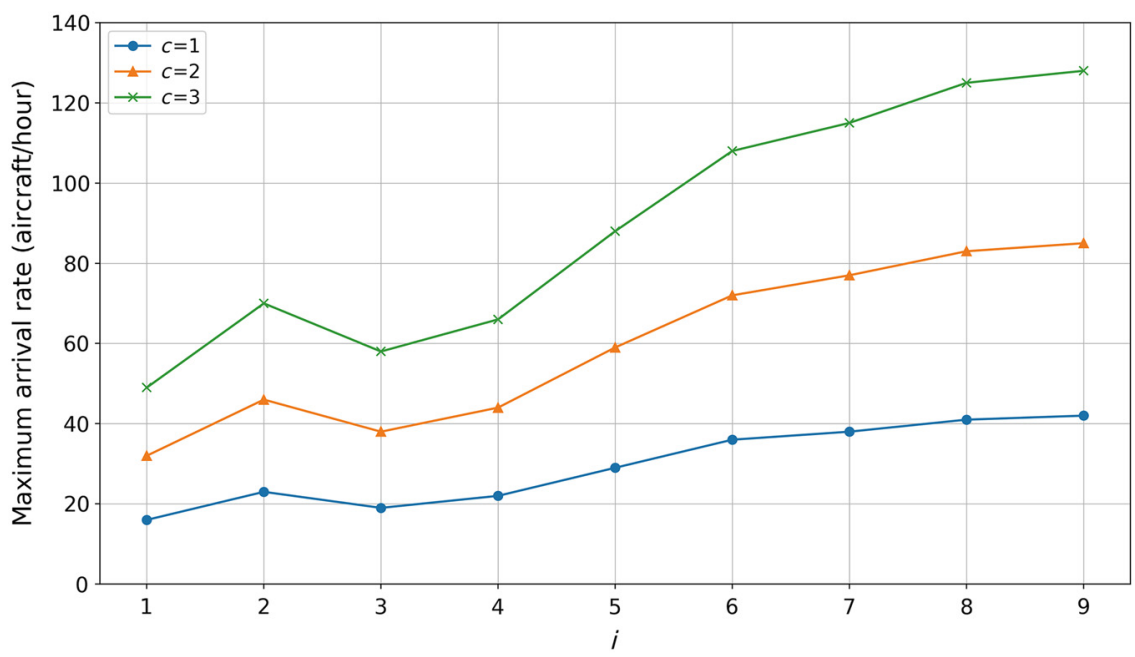

Figure 10. Comparison of maximum arrival rate satisfying $\rho<1$.

Figure 10 shows the estimation of the maximum arrival rate $\lambda_{i}, i \in\{1,2, \ldots, 9\}$, under a fixed service rate $\mathbb{E}\left[B_{i}\right]$ (see Table 6) and airspace capacity $c_{i} \in\{1,2,3\}$, such that $\rho \rightarrow 1$. As shown in Fig. 10, increasing the airspace capacity $c_{i}$ allows for higher arrival rates for all airspace $i \in\{1, \ldots, 9\}$. For $c=1,32$ arrivals per hour is not allowed because the queue stability condition for airspace $i \in\{1,2, \ldots, 5\}$ no longer holds. When $c=2$, the maximum arrival rate allowed in airspace $i=1$ is 32 arrivals, while 40 arrivals are not allowed in airspace $i=3$. If $c=3$, then for all airspace $i \in\{1,2, \ldots, 9\}$ it is possible to have 40 arrivals per hour. Thus, for airspace $i=1$, we need to ensure a capacity of at least $c_{1}=2$ to be able to handle 32 arrivals per hour, while $c_{1}=3$ is required to be able to handle 40 arrivals per hour.

Table 7 summarises the minimum $c_{i}$ values to stabilise the $G / G / c$ queuing model for airspace $i, i \in\{1,2, \ldots, 9\}$. Table 7 shows that the airspace closer to the airport requires more capacity (larges values for $c_{i}$ ), especially in the airspace $i \in\{1,3\}$. This is because close to the destination airport, i.e. airspace $i=1$, the aircraft slow down. Thus, the mean service rate is reduced. In the airspace $i=3$ and 4 , especially $i=3$, the inter-aircraft arrival time is controlled by using vectoring operations. As a result, in this airspace the aircraft change their heading direction and adjust the spacing between succeeding aircraft. Therefore, the service rate $\mu_{i}$ is reduced in the airspace $i=1,3,4$. In $i=2$, the aircraft inter-arrival time is already controlled, and most arriving aircraft follow in-trail because the airspeed is faster than in the $i=1$ airspace.

\subsection{Impact on aircraft arrival delay when increasing airspace capacity}

In this section we estimate the arrival delay time in airspace $i \in\{1,2,3,4\}$, where the maximum arrival rate is limited in comparison with the airspace $i, i \in\{5, \ldots, 9\}$ (see Fig. 10). As shown in Fig. 11, we consider five combinations of arrival rates and airspace capacities, which satisfy the minimum $c_{i}$ in Table 7 , to estimate the arrival time delay. First, for the current arrival rate of 30 arrivals per hour, the capacity $c=2$ results in an increased arrival time delay in comparison with the cases when $c=3$. Thus, increasing the airspace capacity to $c_{i}=3, i \in\{1,2,3,4\}$, is an efficient solution for mitigating arrival delay. However, even when 
Table 7

Minimum $c_{i}$ values that enable the arrival rates of 32, 36, and 40 arrivals per hour, respectively, to be handled in airspace $i, i \in\{1,2, \ldots, 9\}$

\begin{tabular}{llllllllll} 
Arrival rate & \multicolumn{10}{c}{ Airspace area $\boldsymbol{i}$} \\
(aircraft/hour) & $\mathbf{1}$ & $\mathbf{2}$ & $\mathbf{3}$ & $\mathbf{4}$ & $\mathbf{5}$ & $\mathbf{6}$ & $\mathbf{7}$ & $\mathbf{8}$ & $\mathbf{9}$ \\
32 & 2 & 2 & 2 & 2 & 2 & 1 & 1 & 1 & 1 \\
36 & 3 & 2 & 2 & 2 & 2 & 1 & 1 & 1 & 1 \\
40 & 3 & 2 & 3 & 2 & 2 & 2 & 2 & 1 & 1
\end{tabular}

Table 8

Comparison of the mean and STD of the arrival time separation for the metering operation

\section{Current ICAO standard RECAT-Dubai RECAT-EU RECAT-US 1.5}

Runway 22, south wind operation, 5:00PM-10:00PM

$\begin{array}{lllll}\text { Mean (NM) } & 4.430 & 3.858 & 3.869 & 4.061 \\ \text { STD (NM) } & 0.8185 & 0.4197 & 0.4270 & 0.7327\end{array}$

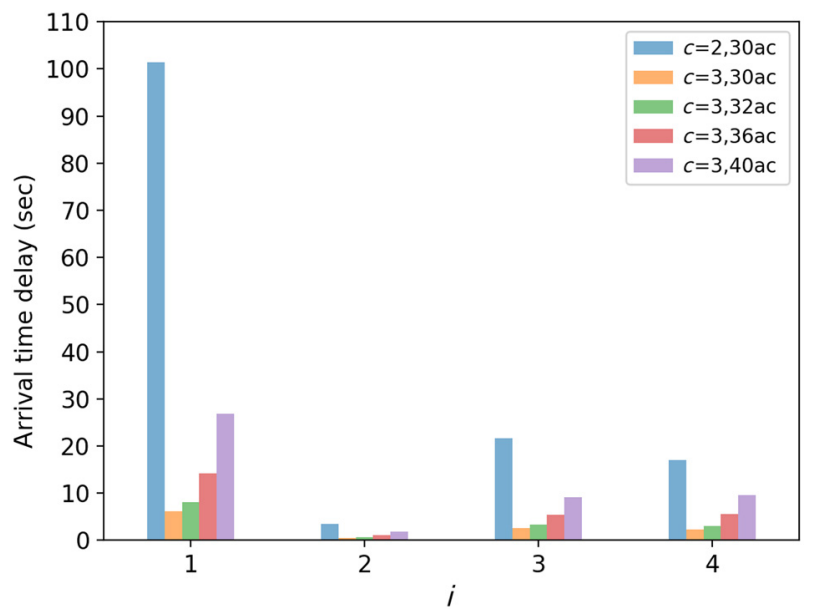

Figure 11. Comparison of arrival delay time in airspace $i \in\{1,2,3,4\}$.

considering $c_{i}=3$, for high arrival rates of 40 aircraft per hour, the arrival delay time remains high, especially in the case of airspace $i=1$.

\subsection{Aircraft arrival delay for various values of the variance of the service time and the variance of the inter-arrival time}

Generally, reducing the variance of the service time and inter-arrival time are options to mitigate the delay in the arrival time in each airspace $i \in\{1,2, \ldots, 9\}$. In this section we determine the arrival delay for various values of the variance of the service time and the inter-arrival 
(a)

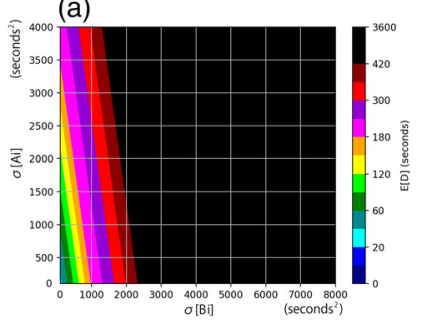

(b)

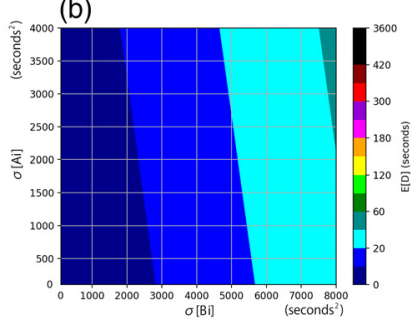

(c)

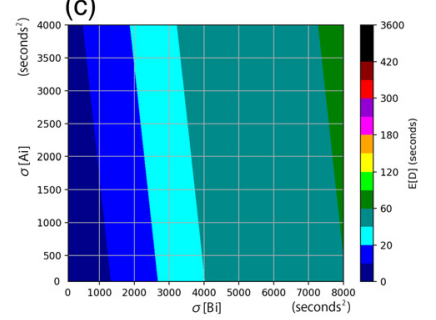

Figure 12. Aircraft arrival delay for various values of the variance of the inter-arrival time and service time - airspace $i=1$. (a) $c_{1}=2,32$ arrivals per hour. (b) $c_{1}=3,32$ arrivals per hour. (c) $c_{1}=3,36$ arrivals per hour.

(a)

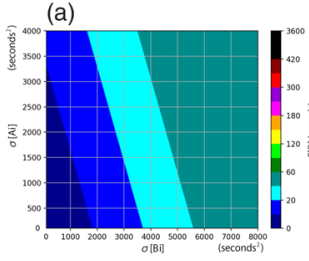

(b)

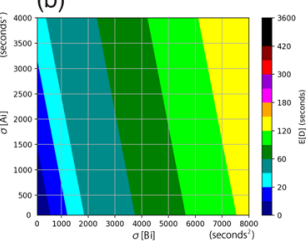

(c)

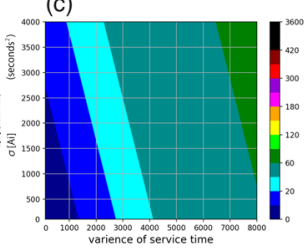

(d)

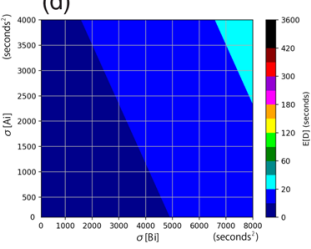

Figure 13. Arrival delay under various values for the variance of the inter-arrival time and service time $c_{i}=2,32$ arrivals per hour, at airspace $i \in\{2,3,4,5\}$. (a) $i=2$. (b) $i=3$. (c) $i=4$. (d) $i=5$.

time, respectively. We consider different aircraft arrival rates and airspace capacities $c_{i}$ such that the requirements in Table 7 are satisfied.

Figure 12 shows the arrival delay for various values of the airspace capacity $c_{1}$ and aircraft arrival rates. Figure 12(a) indicates that, when $c_{1}=2$, the arrival delay dramatically increases even though the variance decreases. Thus, $c_{1}=3$ is necessary at airspace $i=1$ to enable 32 arrivals per hour to be handled (see Fig. 12(b)). Increasing the variance of the service time is critical for the arrival delay, especially when considering higher arrival rates, e.g. 32 or 36 arrivals per hour. In general, the variance of the service time at airspace $i=1$ is larger than that in the other airspace $i, i \in\{2,3, \ldots, 9\}$ (see also Table 6) because in this area the arriving aircraft slow down before landing, and they are also affected by the wind velocity and wind direction associated with the used runway configuration. Because of this, in airspace $i=1$, significant traffic control is necessary to ensure that the arrival delay is limited to the minimum.

Figures 13-15 show the arrival delay by varying the variance of the service time and interarrival time, respectively, at airspace $i \in\{2,3,4,5\}$. We consider the case when $c_{i} \in\{2,3\}$ and an arrival rate of 32 and 36 aircraft arriving per hour. The lowest service rate (the service time with the highest mean) is in airspace $i=3$ among the airspace $i \in\{2,3,4,5\}$ (see Table 6), and the second lowest service rate is in airspace $i=4$ (see Table 6). This can be explained by the fact that currently, in these two areas, the air traffic controllers space the arriving aircraft. Thus, in the airspace $i=3,4$, when $c_{i}=2$, the arrival delay time increases even for small values of the service and inter-arrival variance, as shown in Figs 13 and 14. Increasing the airspace capacity $c_{i}=3$ is the most realistic solution to mitigate the arrival delay time, rather than minimising the variance of the service time and the inter-arrival time (see Fig. 15). 

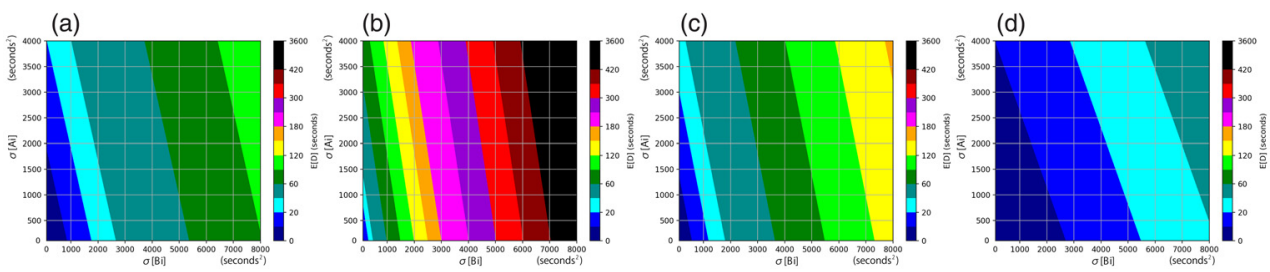

Figure 14. Arrival delay for various values of the variance of the inter-arrival time and the service time, $c_{i}=2,36$ arrivals per hour, at airspace $i \in\{2,3,4,5\}$. (a) $i=2$. (b) $i=3$. (c) $i=4$. (d) $i=5$.
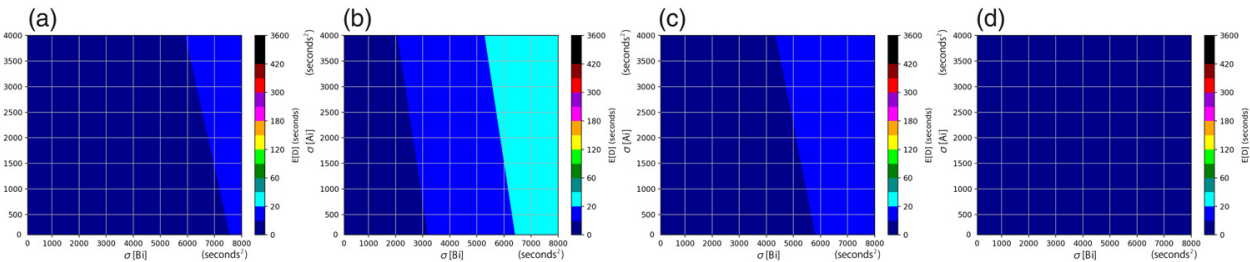

Figure 15. Arrival delay under various values for the variance of the inter-arrival time and the service time, $c_{i}=3,36$ arrivals per hour, at airspace $i \in\{2,3,4,5\}$. (a) $i=2$. (b) $i=3$. (c) $i=4$. (d) $i=5$.

(a)

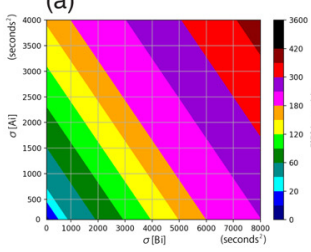

(b)

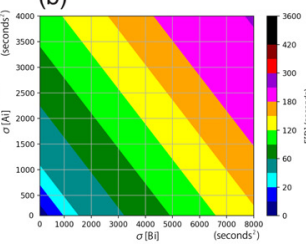

(c)

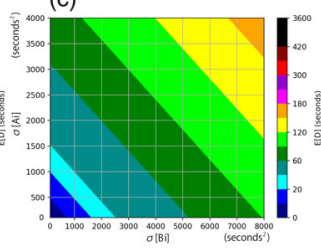

(d)

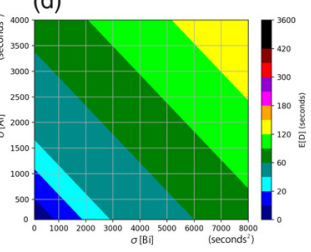

Figure 16. Arrival delay for various values of the variance of the inter-arrival time and the service time, $c_{i}=1,32$ arrivals per hour, at airspace $i \in\{6,7,8,9\}$. (a) $i=6$. (b) $i=7$. (c) $i=8$. (d) $i=9$.

(a)

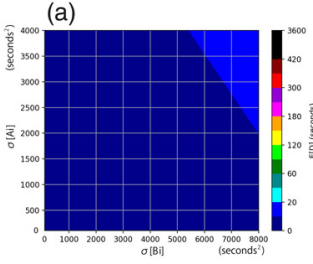

(b)

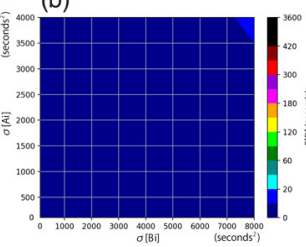

(c)

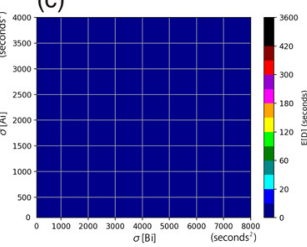

(d)

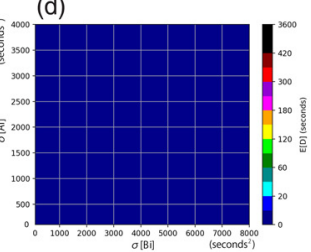

Figure 17. Arrival delay for various values of the variance of the inter-arrival time and the service time, $c_{i}=2,32$ arrivals per hour, at airspace $i \in\{6,7,8,9\}$. (a) $i=6$. (b) $i=7$. (c) $i=8$. (d) $i=9$.

Figures 16-18 show the arrival delay for various values of the variance of the service time and inter-arrival time at airspace $i \in\{6,7,8,9\}$. Although the minimum airspace capacity in these areas is estimated to be $c_{i}=1, i \in\{6,7,8,9\}$, as shown in Table 7 , in the airspace $i \in$ $\{6,7,8,9\}, c_{i}=2$ is required to minimise the arrival delay time for both of the arrival rates of 32 and 36 arrivals per hour. 

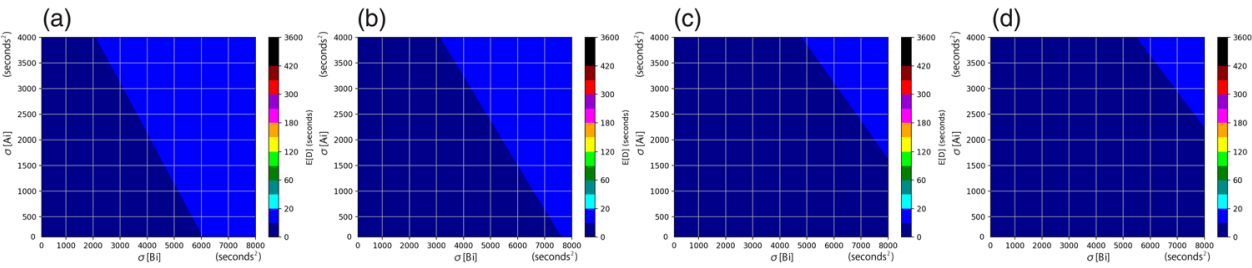

Figure 18. Arrival delay under various values of the variance of the inter-arrival time and the service time, $c_{i}=2,36$ arrivals per hour, at airspace $i \in\{6,7,8,9\}$. (a) $i=6$. (b) $i=7$. (c) $i=8$. (d) $i=9$.

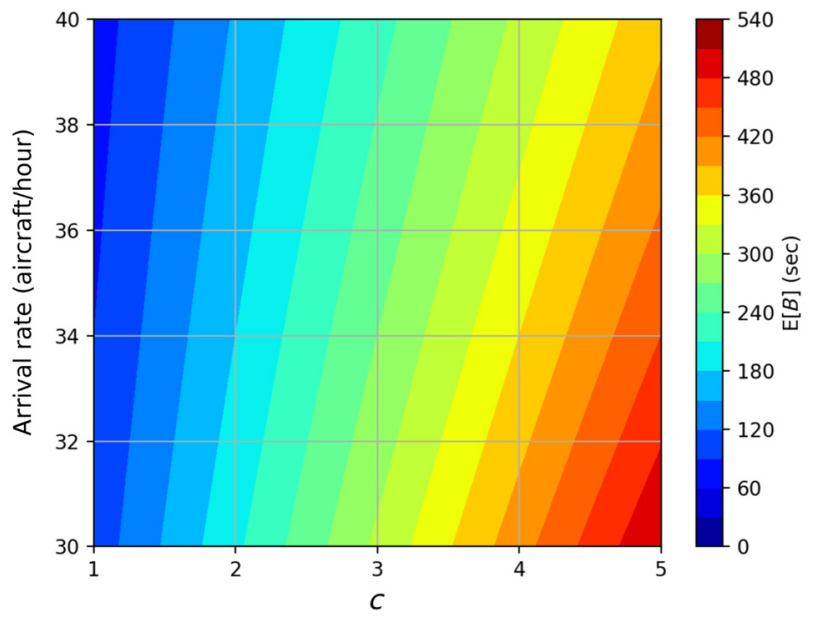

Figure 19. Estimation of service time $\mathbb{E}[B]$.

These results clarify that increasing the airspace capacity is a higher-priority strategy than the effort of minimising the variance of the service time, except for the case of airspace $i=5$. Figure 14(d) shows that, for the current inter-arrival time with a variance of 3,488 $\sec ^{2}$ (see Table 6) the arrival delay time is large. This shows that for airspace $i=5$, to limit the arrival delay we have two possible strategies when considering an arrival rate of 36 arrivals per hour: (1) increase the airspace capacity $c_{5}=3$ (see Fig. $15(\mathrm{~d})$ ) or, (2) maintain $c_{5}=2$ and limit the inter-arrival time variance (see Fig. 14(d)).

\subsection{Service time envelopes estimating the arrival delay}

Optimising the service time in each airspace $i$ is the other potential solution for mitigating the arrival delay time while increasing the arrival rate. Figure 19 shows the estimated envelope of the maximum service time satisfying the condition $\rho<0.85$. Figure 19 shows that an increase in the arrival rate reduces the service time at the same capacity $c$. At a larger capacity $c$, the service time is increased.

The estimated service time shown in Fig. 19 is given to airspace $i \in\{1,2, \ldots, 9\}$ for estimating the arrival time delay corresponding to airspace capacity $c_{i}$ and the arrival rate as shown in Fig. 20. One of the features clarifies that the arrival time delay increases while $i$ increases if the same amount of service time is allowed. This means that the delay in the arrival time increases if the same amount of flight time associated with the airspace closer 


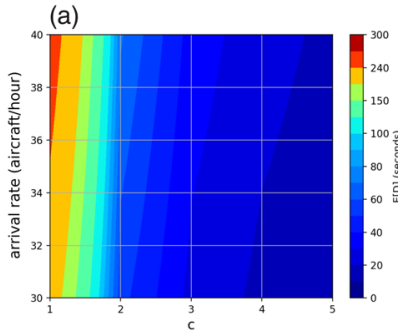

(d)

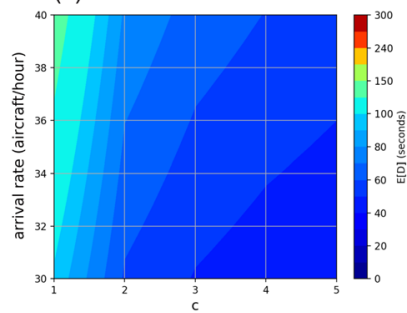

(g)

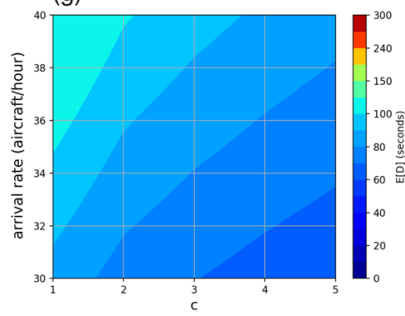

(b)

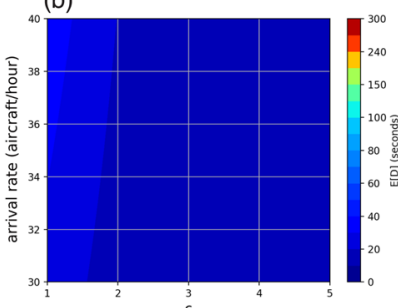

(e)

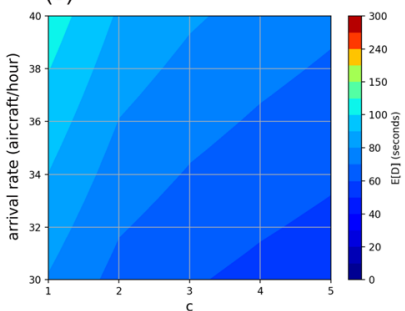

(h)

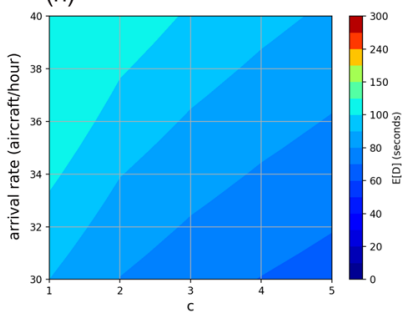

(c)

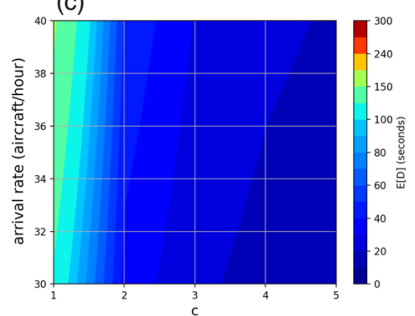

(f)

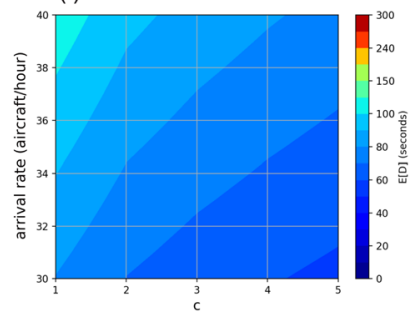

(i)

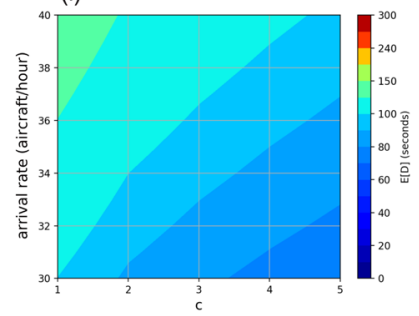

Figure 20. Estimating the impact of the service time at airspace $i \in\{1,2,3, \ldots, 9\}$. (a) $i=1$. (b) $i=2$. (c) $i=3$. (d) $i=4$. (e) $i=5$. (f) $i=6$. (g) $i=7$. (h) $i=8$. (i) $i=9$.

to the arrival airport would be associated with the airspace farther away. In other words, it is more efficient to extend the flight time closer to the arrival airport than in the airspace farther away. One of the reasons is that the variance of the inter-arrival time becomes larger in the case of the airspace farther away, as shown in Table 6. To increase the service time is more effective when the variance of the inter-arrival time is smaller. This means that extending the flight time in airspace farther away is not the solution to reduce the arrival delay time.

\subsection{DISCUSSION}

The results of our analysis clarified that the best strategy to increase the amount of arriving traffic while minimising the arrival delay time in the airspace within a circle with a radius of $100 \mathrm{NM}$ centered at the Tokyo International Airport is as follows: (1) allowing an airspace capacity $c_{1}=3$ and minimising the variances of the service time in the airspace $i=1 ;(2)$ allowing $c_{i}=3$ in the airspace $i \in\{2,3,4\}$; (3) allowing $c_{5}=3$, or $c_{5}=2$ and reducing the variances of the inter-arrival time; (4) allowing $c_{i}=2$ in the airspace $i \in\{6,7,8,9\}$. The airspace $i=5,6$ is the transition area in which to control the airspace capacity from $c_{i}=2$ to $c_{i}=3$. These results provide the key requirements for designing the E-AMAN system intended to support the arrival scheduling tasks of air traffic controllers. 


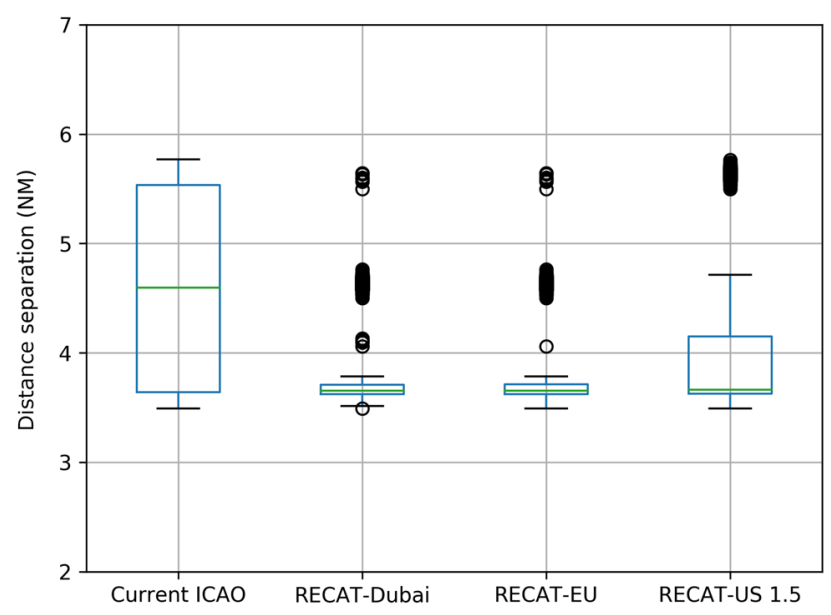

Figure 21. Comparison of the distance separation with the safety margin for the metering condition of different standards.

Satisfying $2<c_{i} \leq 3$ means that in-trail arrival aircraft achieve an approximate distanceseparation of between 3.3 and 5NM. Considering the queue of preceding arrival aircraft, the preferred distance-separation should be closer to 3.3NM. Figure 21 and Table 8 compare the mean and STD of the distance-separation each of the wake vortex categories achieves at Runway 22 with the safety margin of the metering operation (see Table 5). A comparison of the mean values of the distance-separation indicates that all four standards satisfy $2<c_{i} \leq$ 3 . However, 36 arrivals per hour would be achievable only by applying RECAT-Dubai and RECAT-EU at Runway 22 (see Fig. 7).

In the current operation, radar separation minima is $3 \mathrm{NM}$ in the terminal area, and $5 \mathrm{NM}$ in the en-route sectors. Currently, the boundary between the terminal and en-route area for the south-west traffic is located at around $60-80 \mathrm{NM}$ away from the Tokyo International Airport. Therefore, the recommendations above satisfy the current operational constraints.

Shifting the arrival delay time to airspace farther away from the arrival airport is not an option to mitigate the arrival delay time because the variances of the inter-arrival time increase as the distance from the arrival airport becomes longer. Extending the mean of the service time (flight time) only has a powerful effect when the aircraft inter-arrival time is controlled to minimise the variance. Thus, arrival strategies to extend the aircraft flight time, i.e. vectoring and point-merge, are effective in the airspace close to the airport where the variance of the inter-arrival time is controlled.

\subsection{CONCLUSION}

This study has analysed arrival strategies to minimise the delay in aircraft arrival while allowing future growth in the amount of arrival traffic. A data-driven queuing model was proposed to analyse the impacts of applying new wake vortex minima, which reduces the aircraft interarrival time, on the traffic flow of aircraft arriving within a circle with a radius of $100 \mathrm{NM}$ centered at the arrival airport. Two years of data consisting of radar tracks and flight plans of air traffic arriving at Tokyo International Airport were analysed as a case study. The volume of future arrival traffic was estimated by using both the new time-separation standards and 
the level of automation support. Based on the data-driven analysis and the estimation of the future traffic volume, a queue-based model was proposed to analyse the impact on the delay in arrival time of potential future arrival strategies. Based on the analysis, the best arrival strategy was suggested based on the distance from the arrival airport.

Based on the proposed requirements for future arrival scheduling, the next step would be the engineering design of the E-AMAN system. Human-in-the-loop simulation, which evaluates the operational feasibility of air traffic controllers and pilot operation, will be conducted to clarify the effectiveness of the proposed design requirements in the real operation.

In this study, the volume of traffic arriving in future has been estimated by only considering aircraft separation standards and the performance of automation support for arrival management. However, this estimation would be more precise if we were to consider constraints on the departure and airport management, i.e. runway occupancy time. In future, we plan to extend the proposed approach not only to arrival traffic, but also departure traffic and the airport surface movements.

\section{ACKNOWLEDGEMENTS}

This research was conducted under CARATS initiatives supported by the Japan Civil Aviation Bureau (JCAB), Ministry of Land, Infrastructure, Transport and Tourism (MLIT) as the 'Studies on the Extended Arrival Management.' This research was also supported by the Ministry of Education, Culture, Sports, Science and Technology (MEXT) as the 'PostK Computer Exploratory Challenge' (Exploratory Challenge 2: Construction of Models for Interaction Among Multiple Socioeconomic Phenomena, Model Development and its Applications for Enabling Robust and Optimized Social Transportation Systems, Project ID: hp180188). The authors are grateful to JCAB for providing air traffic data, and Ms. Mayumi Ohnuki for assisting data preparation and figure corrections. The authors would like to thank Enago (www.enago.jp) for the English language review.

\section{REFERENCES}

1. Rooseleer, F. and TREVE, V. RECAT-EU European wake turbulence categorisation and separation minima on approach and departure. Eurocontrol, 2015. https://www.eurocontrol.int/publication/ european-wake-turbulence-categorisation-and-separation-minima-approach-and-departure

2. Federal Aviation Administration (FAA). Wake turbulence recategorization, Air Traffic Organization Policy, Order: JO 7110.659B, 2015.

3. General Civil Aviation Authority. OMDB - Dubai/international - implementation of Dubai recategorisation (RECAT) wake turbulence separation scheme within Dubai CTA, AIP Supplement - United Arab Emirates, 2019.

4. Passenger traffic 2016 final (annual), Airports Council International (ACI), 1st January, 2018.

5. ERzBerger, H. and IтOH, E. Design principles and algorithms for air traffic arrival scheduling, NASA/TP-2014-218302, 2014.

6. Federal Aviation Administration. Nextgen portfolio - time based flow management, 2017.

7. Thipphavong, J., Jung, J., Swenson, H., Martin, L., Lin, M. and NGuYen, J. Evaluation of the terminal sequencing and spacing system for performance-based navigation arrivals, 2013 IEEE/AIAA 32nd Digital Avionics Systems Conference (DASC), 2013.

8. VAN TulD, P. Flight deck interval management flight test final report, NASA/CR-2017-219626, June 2017, 2017.

9. European Commission. Cross border SESAR trials for enhanced arrival management: periodic reporting for period 1 - PJ25 XSTREAM, 2017. 
10. International Civil Aviation Organization. Long range ATFM concept trials, The 8th Meeting of the ICAO Asia/Pacific Air Traffic Flow Management Steering Group (ATFMSG/8), May 2018, 2018.

11. Itoh, E., Brown, M., Senoguchi, A., Wickramasinghe, N. and Fukushima, S. Future arrival management collaborating with trajectory-based operations. In Air Traffic Management and Systems II, pp 137-156, Springer, Japan, 2017.

12. BäUerLe, N., ENGELHARDT-FunK, O. and Kolonko, M. On the waiting time of arriving aircrafts and the capacity of airports with one or two runways, Eur J Oper Res, 2007, 177, (2), pp 1180-1196.

13. Long, D., Johnson, J., GaIER, E.M. and KostiuK, P.F. Modeling air traffic management technologies with a queuing network model of the national airspace system, NASA Langley Technical Report Server, 1999.

14. Rue, R.C. and Rosenshine, M. The application of semi-Markov decision processes to queuing of aircraft for landing at an airport, Transp Sci, 1985, 19, (2), pp 154-172.

15. BOLENDER, M.A. and SLATER, G.L. Evaluation of scheduling methods for multiple runways, J Aircr, 2000, 37, (3), pp 410-416.

16. Iтон, E. and Mıтісі, M. Queue-based modeling of the aircraft arrival process at a single airport, Aerospace, 2019, 6, (10), 103. https://doi.org/10.3390/aerospace6100103.

17. Ітон, Е. and Mıтісі, M. Analyzing tactical control strategies for aircraft arrivals at an airport using queue-based modeling, Under Review, 2019.

18. International Civil Aviation Organization. Long-term traffic forecasts passenger and cargo, total passenger traffic: history and forecasts, $\mathrm{p} 10,2016$.

19. ARbuckle, D. Interval management application, ICAO Aircraft Surveillance Applications Workshop, March 2017.

20. Whitт, W. The queuing network analyzer, Bell Syst Tech J, 1983, 62, (9), pp 2779-2815.

21. Whitt, W. Approximations for the GI/G/m queue, Prod Oper Manage, 1993, 2, (2), pp 114-161.

22. Kimura, T. A two-moment approximation for the mean waiting time in the GI/G/s queue, Manage Sci, 1986, 32, (6), pp 751-763.

23. Kingman, J.F.C. The single server queue in heavy traffic, In Mathematical Proceedings of the Cambridge Philosophical Society, vol 57, pp 902-904, Cambridge University Press, UK, 1961.

24. Hillier, F. Introduction to Operations Research, Tata McGraw-Hill Education, US, 2012.

25. AdAn, I. and ResING, J. Queuing systems, Eindhoven University, 2015. 